\title{
Surface Diffusion Anomaly near a Substrate Phase Transition: H on W(100)
}

\author{
Lei Cai, ${ }^{1}$ M. S. Altman, ${ }^{1}$ E. Granato, ${ }^{2}$ T. Ala-Nissila, ${ }^{3,4}$ S. C. Ying, ${ }^{3}$ and Xudong Xiao ${ }^{1, *}$ \\ ${ }^{1}$ Department of Physics, The Hong Kong University of Science and Technology, Hong Kong, SAR, China \\ ${ }^{2}$ Instituto Nacional de Pesquisas Espaciais, Sao Jose dos Campos, SP Brazil \\ ${ }^{3}$ Department of Physics, Brown University, Providence, Rhode Island 02912 \\ ${ }^{4}$ Helsinki Institute of Physics and Laboratory of Physics, Helsinki University of Technology, \\ P.O. Box 1100, FIN-02015 HUT, Espoo, Finland
}

(Received 25 January 2002; published 20 May 2002)

\begin{abstract}
Using a linear optical diffraction method, we have experimentally studied the long predicted diffusion anomalous behavior for $\mathrm{H} / \mathrm{W}(100)$ near the reconstructive phase transition of the $\mathrm{W}(100)$ substrate. This anomaly manifests itself in the form of a strong dip in the diffusion coefficient $D$ at the transition temperature $T_{C}$. We interpret the strong reduction of $D$ as a result of the diverging friction damping near the transition. The finite dip in $D$ instead of a vanishing $D$ at $T_{C}$ also demonstrates the importance of the non-Markovian (memory) deviation from the simple instantaneous damping picture.
\end{abstract}

DOI: $10.1103 /$ PhysRevLett.88.226105

Surface diffusion near an adsorbate or substrate phase transition is expected to have anomalous, non-Arrhenius temperature dependence based on theoretical grounds [1-3]. Experimentally, such behavior has often been observed near adsorbate layer phase transitions and is sometimes even used to identify surface phase transitions [4-6]. However, the underlying physics for such observations is rarely understood, mostly due to the lack of knowledge of the nature of the relevant phase transitions. In contrast, the nature of the substrate reconstructive phase transition of the W(100) surface is well understood [7-16]. For the continuous surface reconstruction, there also exist clear theoretical predictions for the anomaly of the diffusion coefficient $D$ [1]. Thus, it provides an ideal model system to investigate the effect of the substrate phase transition on surface diffusion.

In this work, we set out to investigate experimentally the predicted diffusion anomaly of $\mathrm{H} / \mathrm{W}(100)$ near the substrate reconstructive phase transition. The experiments were carried out in a UHV system with a base pressure of $2 \times 10^{-10}$ torr. A single crystalline W(100) sample was cut and mechanically polished to within $0.1^{\circ}$ from the (100) plane. This substrate was first cleaned by cycles of oxygen treatment at $2 \times 10^{-7}$ torr and $1480 \mathrm{~K}$ with subsequent flashings to $2000 \mathrm{~K}$, and then recleaned by flashing to $2000 \mathrm{~K}$ prior to each diffusion measurement. Fast liquid nitrogen cooling from 2000 to $90 \mathrm{~K}$ could be achieved in about $3 \mathrm{~min}$. The dissociative adsorption of $\mathrm{H}$ on the $\mathrm{W}(100)$ surface was carried out at approximately $90 \mathrm{~K}$ by leaking $\mathrm{H}_{2}$ gas into the chamber followed by annealing for 2 min at room temperature [10], and the $\mathrm{H}$ coverage was calibrated by thermal desorption spectroscopy. The diffusion coefficient $D$ was measured using the linear optical diffraction technique [17] with a small coverage modulation of about 0.03 ML. $D$ was measured as a function of substrate temperature, $T$, over a range from 210 to $430 \mathrm{~K}$ for three different $\mathrm{H}$ coverages, $0.08,0.17$, and 1.2 ML. The adsorbate grating spacing was chosen to be
PACS numbers: 68.43.Jk, 68.35.Fx, 68.35.Rh, 68.47.De

$3.9 \mu \mathrm{m}$ when $D$ is slower than $\sim 10^{-9} \mathrm{~cm}^{2} / \mathrm{s}$ and $6.7 \mu \mathrm{m}$ when $D$ is faster than $\sim 10^{-9} \mathrm{~cm}^{2} / \mathrm{s}$. At the overlapping temperatures, the diffusion coefficients measured by both grating spacings were the same. From the diffusion measurements along different azimuthal orientations (rotated by about $45^{\circ}$ ) on two different samples, both with $<0.1^{\circ}$ miscut, we observed no azimuthal angle dependence for the present data.

The clean $\mathrm{W}(100)$ surface undergoes a second order phase transition from a high temperature $(1 \times 1)$ to a low temperature $c(2 \times 2)$ phase of $p 2 m g$ symmetry at a transition temperature $T_{C} \sim 250 \mathrm{~K}[10,11]$. At very low $\mathrm{H}$ coverages $(<0.12 \mathrm{ML})$, the nature of the phase transition is similar to that of the clean surface with $T_{C}$ increasing steadily with $\mathrm{H}$ coverage $[10,14]$. Above $0.12 \mathrm{ML}$, the low temperature phase switches to a new $c(2 \times 2)$ structure with $\mathrm{c} 2 \mathrm{~mm}$ symmetry $[10,13]$. The phase diagram of the $\mathrm{W}(100)$ reconstructive transition has been well documented [10] and is shown in Fig. 1a. To verify this phase diagram and also obtain an independent calibration for the coverage, we first studied the transition with low energy electron diffraction (LEED). In Fig. 1b, the normalized peak intensity of the $(1 / 2,1 / 2)$ LEED spot is plotted as a function of temperature for the clean surface, 0.08 and $0.17 \mathrm{ML}$ of $\mathrm{H}$ coverages. The inset also shows the typical LEED patterns at high and low temperatures, indicating the good ordering of the substrate. From the half intensity points, indicated by the horizontal dashed line in Fig. 1b, we estimate the transition temperatures for clean, 0.08 and 0.17 ML H coverage surfaces to be 245, 305, and $345 \mathrm{~K}$, respectively, in agreement with the phase diagram in Fig. 1a.

In Fig. 2, the diffusion results for 0.08, 0.17, and 1.2 ML $\mathrm{H}$ coverage on $\mathrm{W}(100)$ are shown as a function of the reciprocal temperature. At $0.08 \mathrm{ML}$, the data at low temperatures $(<270 \mathrm{~K})$ can be fitted by the Arrhenius behavior $D=D_{0} \exp \left(-E_{\mathrm{diff}} / k_{B} T\right)$, with a prefactor $D_{0} \sim$ $10^{-(1.9 \pm 0.2)} \mathrm{cm}^{2} / \mathrm{s}$ and a diffusion activation energy 

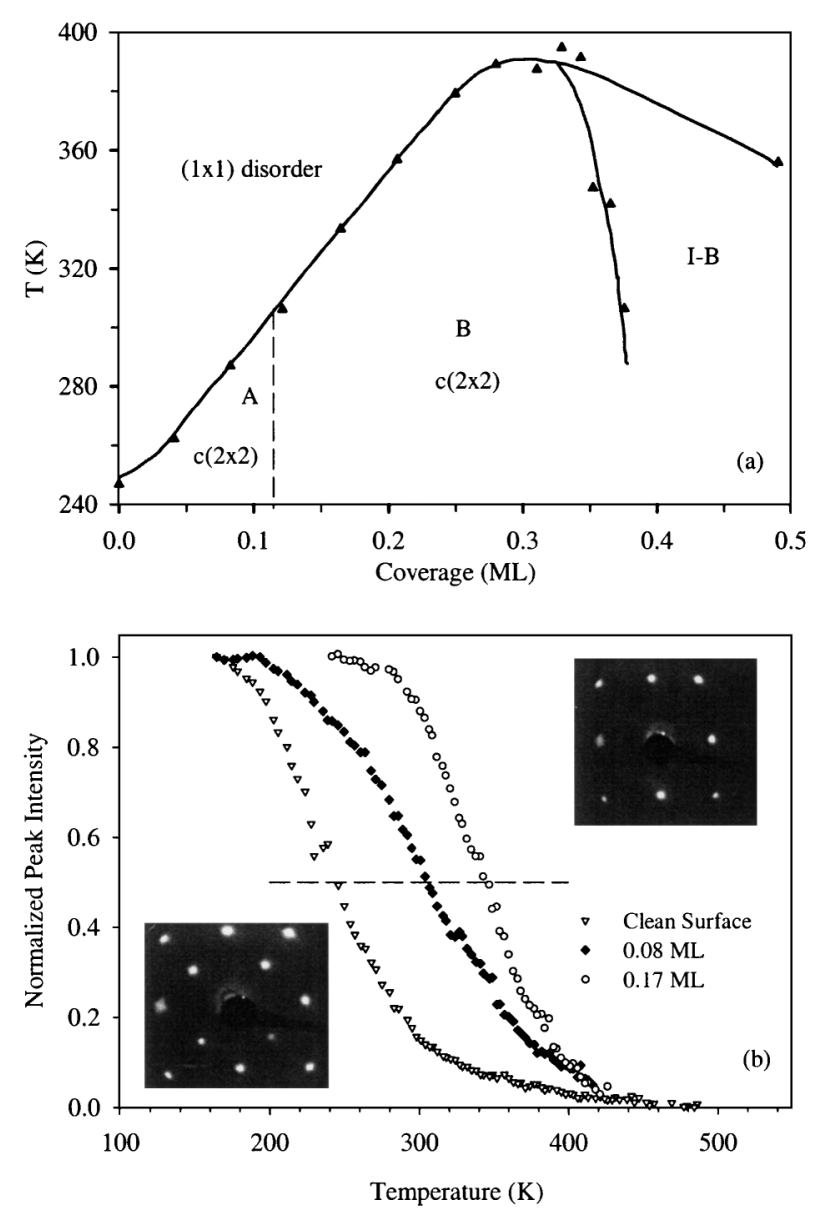

FIG. 1. (a) Phase diagram of the $\mathrm{H} / \mathrm{W}(100)$ system taken from Ref. [10], where $A$ is the $c(2 \times 2)$ phase with $p 2 m g$ symmetry, $B$ is the $c(2 \times 2)$ phase with $c 2 m m$ symmetry and $I-B$ is an incommensurate phase of $B$. (b) Normalized LEED intensity of the $(1 / 2,1 / 2)$ spot as a function of temperature for the clean, 0.08 and 0.17 ML $H$ covered $\mathrm{W}(100)$ surface. The insets show the $(1 \times 1)$ and $c(2 \times 2)$ LEED patterns. The dashed line indicates the half intensity points used to estimate the transition temperature.

$E_{\text {diff }} \sim 10.8 \pm 1.4 \mathrm{kcal} / \mathrm{mol}$. However, above $270 \mathrm{~K}$ the diffusion coefficient deviates significantly from the Arrhenius behavior and shows a pronounced dip around $290 \mathrm{~K}$. The behavior of $D$ for $0.17 \mathrm{ML} \mathrm{H}$ coverage is similar to that for $0.08 \mathrm{ML}$. Below $325 \mathrm{~K}$, the data obey an Arrhenius behavior, but there is again a strong anomaly with a pronounced dip of $D$ occurring at a higher temperature near $355 \mathrm{~K}$. Finally, for the coverage of $1.2 \mathrm{ML}$, there is no observable diffusion anomaly (Fig. 2 inset). The diffusion data at this coverage can be fitted to an Arrhenius behavior over the entire temperature range with $D_{0} \sim$ $10^{-(2.2 \pm 0.2)} \mathrm{cm}^{2} / \mathrm{s}$ and $E_{\mathrm{diff}} \sim 10.5 \pm 0.3 \mathrm{kcal} / \mathrm{mol}$. When compared to the phase diagram in Fig. 1a and the LEED result in Fig. 1b, we see that the temperatures at which the diffusion anomaly occurs for 0.08 and 0.17 ML correlate with the transition temperatures for the $(1 \times 1)$ to $c(2 \times 2)$ transition at the respective coverages. Furthermore, it is known from previous studies that there is no observable phase transition at 1.2 ML [18].

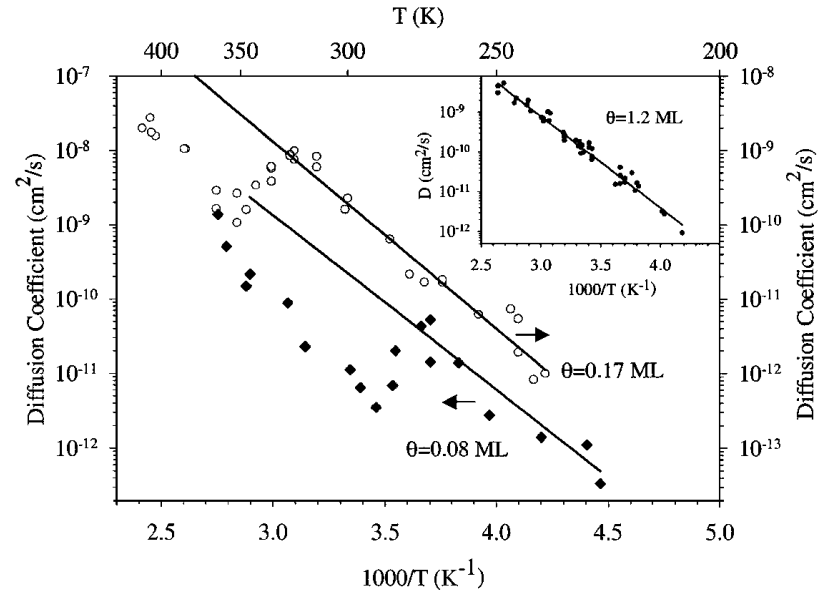

FIG. 2. Diffusion coefficients of $\mathrm{H}$ on $\mathrm{W}(100)$ versus the reciprocal temperature at $0.08(\diamond), 0.17(\circ)$, and $1.2(\bullet$, inset) ML $\mathrm{H}$ coverages. Note that the vertical axes for the different coverages are shifted for clarity. The solid lines are fits to the Arrhenius law.

Correspondingly, our diffusion data at this high coverage show no anomaly at all. The strong correlation of our diffusion data with the phase diagram suggests that the diffusion anomaly observed here is associated with the underlying substrate reconstructive phase transition.

We discuss now the origin of the anomalous diffusion near the substrate phase transition temperature. First, the diffusion energy barrier is determined by the short-range order of the substrate, which does not change abruptly near the phase transition. The prefactor $D_{0}$, on the other hand, is governed by the frictional force on the adatom through its interaction with substrate excitations. At present, there are no reliable estimates for the H/W(100) system regarding the relative dominance of the electronic and vibrational contributions to the friction. However, near the reconstructive phase transition of $\mathrm{W}(100)$, the electronic excitations basically do not change because they are determined by the short-range order, while the vibrational excitations are known to develop anomalous temperature dependence due to critical fluctuations of long wavelengths $[19,20]$. In particular, the modes near the critical wave vector $\mathbf{q}_{0}=(\pi / a, \pi / a)$ soften and become overdamped as $T$ approaches $T_{C}$. The anomalous temperature dependence of the substrate phonons leads to critical singularities in the dynamic structure factor $S(\mathbf{q}, \omega)$ near $T_{C}$ and ultimately to the diffusion anomaly. Near $T_{C}, S(\mathbf{q}, \omega)$ obeys the scaling form $\xi_{c}^{z+(\gamma / v)} g_{ \pm}\left(\left|\mathbf{q}-\mathbf{q}_{0}\right| \xi_{c}, \omega \xi_{c}^{z}\right)$ where $g_{ \pm}$ is a scaling function, $\xi_{c} \propto\left|T / T_{c}-1\right|^{-\nu}$ is the divergent correlation length, $\gamma$ is the susceptibility exponent, and $z$ is the dynamical critical exponent [3]. In the Markovian limit of instantaneous damping, the frictional damping $\eta$ is simply determined by the integration of $S(\mathbf{q}, \omega=0)$ over a range of $\mathbf{q}$ determined by the coupling potential of the $\mathrm{H}$ adatom to the substrate $[1,3]$. This leads then to a $\eta$ diverging as $\left|T-T_{C}\right|^{-x}$ with $x=\nu(z-d)+\gamma$ (in $d$ dimensions) and $D \sim \eta^{-1}$ vanishing as $T$ approaches $T_{C}$. A recent molecular dynamics simulation [3] yields 
a value of the exponent $x=1.8(4)$ for the $\mathrm{H} / \mathrm{W}(100)$ system in the zero coverage limit. We have fitted the present data for $D$ to a power law behavior $\left|T-T_{C}\right|^{x}$ in Figs. $3 \mathrm{a}$ and $3 \mathrm{~b}$. The fit in the critical region yields a value of the exponent $x=1.6(3)$ for $0.08 \mathrm{ML}$ and 1.3(2) for $0.17 \mathrm{ML}$. The value at $0.08 \mathrm{ML}$ is consistent with the theoretical result [3]. This exponent is nonuniversal and its value is expected to depend weakly on the hydrogen coverage which modifies the fourfold anisotropy in the model Hamiltonian [21].

The simple argument presented above applies only when $T$ is not too close to $T_{C}$. Two additional factors need to be considered which kill the infinite divergence of the frictional damping and result in a finite dip in $D$ instead of it vanishing at $T_{C}$. The first is the breakdown of the Markovian approximation that the frictional damping is proportional to the instantaneous velocity $[22,23]$. As one approaches $T_{C}$, the dynamic structure factor $S(\mathbf{q}, \omega)$ get narrower and stronger and develops into a "central peak" structure [3]. When the width of this central peak gets narrower than the characteristic frequency for the motion of the $\mathrm{H}$ adatom, the time scale of the relevant substrate excitations is longer than the time scale of the motion of the hydrogen adatom. At this point, the instantaneous damping picture breaks down, and the divergence in the frictional
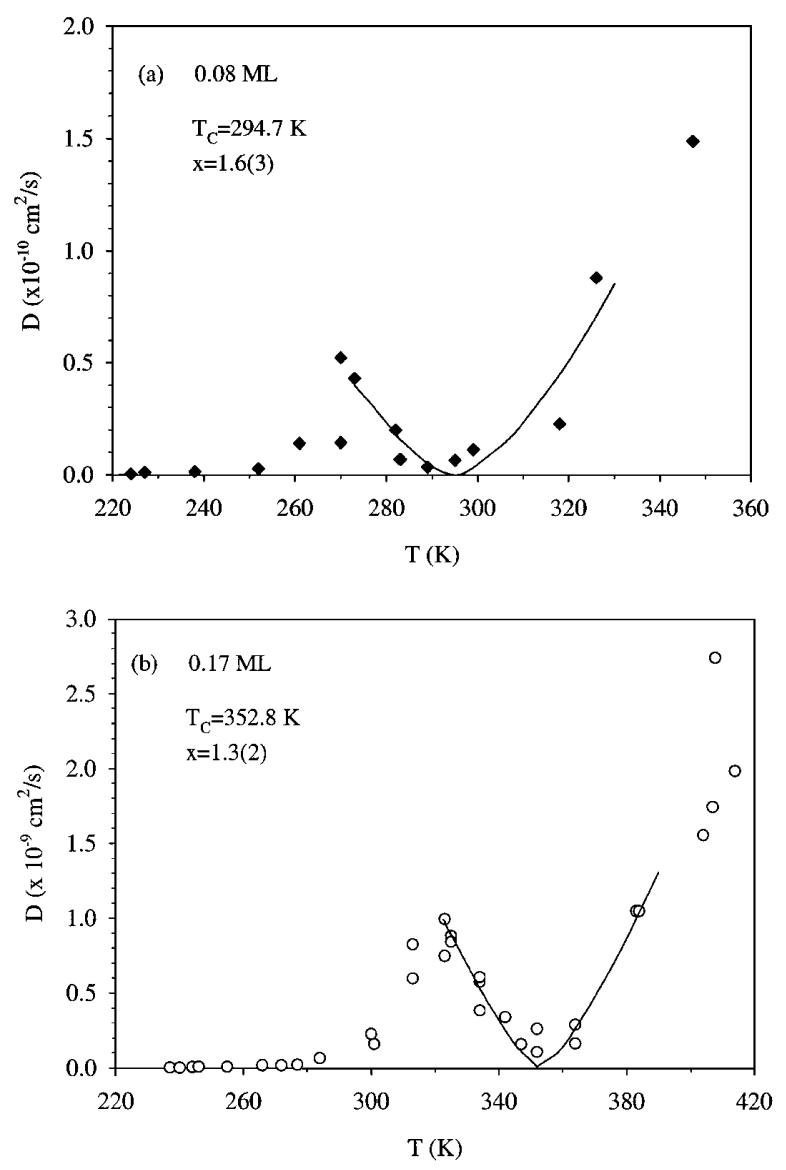

FIG. 3. Fits of $D$ to a power law behavior $\left|T-T_{C}\right|^{x}$ for (a) 0.08 and (b) $0.17 \mathrm{ML} \mathrm{H}$ coverages in the critical region. The fits give $x=1.6(3)$ for $0.08 \mathrm{ML}$ and 1.3(2) for 0.17 ML. damping is cut off. The second factor that suppresses divergence is sample imperfection such as finite size or a finite step density. As one approaches $T_{C}$, the divergence of the correlation length $\xi$ is cut off when it first reaches either the size of the system or the average spacing $L$ between the steps [24]. From the sample miscut angle, we estimate that, for our sample, $L=300 a$ ( $a$ : lattice spacing), leading us well into the critical region before the divergence is finally cut off. In a previous study of diffusion for $\mathrm{H} / \mathrm{W}(100)$ by the field emission fluctuation method [25], it showed no anomaly in the temperature range from 220 and $350 \mathrm{~K}$ for coverages from 0.12 to $1.2 \mathrm{ML}$. This could be due to the suppression of the critical fluctuations associated with the transition either by the small sample size and/or by the strong electric fields in the field emission tip. Away from the critical region, our data agree closely within experimental uncertainty with those from the field emission study at the corresponding coverages [26]. It should be noted that the field emission method, just as the He scattering technique [27], focuses on the microscopic mobility in a small area on the sample and does not feel the effect of the steps directly. So the agreement between the two sets of data indicates that the steps in our sample has little effect on the diffusion except in cutting off the true divergence of the friction near the critical region.

In conclusion, we have observed here the long predicted diffusion anomaly for $\mathrm{W}(100)$ near the reconstructive phase transition of the $\mathrm{W}(100)$ substrate. It manifests itself in the form of a strong dip in the diffusion coefficient at the transition temperature $T_{C}$. The data correlate very well with the previously established phase diagram for this system. It also demonstrates the breakdown of the instantaneous damping picture. This leads to a cutoff of the critical divergence and a transformation of the anomaly from a vanishing of $D$ into a finite dip at $T_{C}$. Finally, we should mention that a very similar anomaly has been observed in the dynamics of the vortex lattice in type II superconductors [28,29]. There, the electrical conductance of the superconductor (inversely proportional to the diffusion constant of the vortex lattice) shows a strong peak at the melting transition of the vortex lattice, yielding the so-called peak effect.

We thank Mr. K.L. Man and Mr. Chaozhi Zheng for their help during the experiment. We acknowledge financial support from the Research Grant Council of Hong Kong through Grant No. HKUST6114/98P, and the William Mong Solid State Cluster Laboratory (L.C., M.S.A., and X.X.), $\mathrm{CNPq}$ and NSF grants (S.C. Y., E. G., AND T. A.-N.), FAPESP (E. G.), and the Academy of Finland through its Center of Excellence program (T. A.-N.).

*To whom correspondence should be addressed. Email address: phxudong@ust.hk

[1] T. Ala-Nissila, W. K. Han, and S. C. Ying, Phys. Rev. Lett. 68, 1866 (1992). 
[2] I. Vattulainen, J. Merikoski, T. Ala-Nissila, and S. C. Ying, Phys. Rev. Lett. 79, 257 (1997).

[3] M. R. Baldan, E. Granato, and S. C. Ying, Phys. Rev. B 62, 2146 (2000).

[4] R. Gomer, Rep. Prog. Phys. 53, 917 (1990), and references therein.

[5] A. G. Naumovets, V. V. Poplavsky, and Yu. S. Vedula, Surf. Sci. 200, 321 (1988).

[6] S. C. Wong and R. Gomer, J. Chem. Phys. 83, 4193 (1985).

[7] K. Yonehara and L. D. Schmidt, Surf. Sci. 25, 238 (1971).

[8] T. E. Felter, R. A. Barker, and P. J. Estrup, Phys. Rev. Lett. 38, 1128 (1977).

[9] M. K. Debe and D. A. King, Phys. Rev. Lett. 39, 708 (1977).

[10] R. A. Barker and P. J. Estrup, J. Chem. Phys. 74, 1442 (1981).

[11] J.F. Wendelken and G. C. Wang, Phys. Rev. B 32, 7542 (1985).

[12] R. A. Barker, P. J. Estrup, F. Jona, and P. M. Marcus, Solid State Commun. 25, 375 (1978).

[13] D. A. King and G. Thomas, Surf. Sci. 92, 201 (1980).

[14] K. Griffiths, D. A. King, and G. Thomas, Vacuum 31, 671 (1981).

[15] C. L. Fu and A. J. Freeman, Phys. Rev. B 37, 2685 (1988).

[16] I. K. Robinson, A. A. MacDowell, M. S. Altman, P. J. Estrup, K. Evans-Lutterodt, J. D. Brock, and R. J. Birgeneau, Phys. Rev. Lett. 62, 1294 (1989).

[17] X. D. Xiao, Y. Xie, and Y. R. Shen, Surf. Sci. 271, 295 (1992).
[18] J. J. Arrecis, Y. J. Chabal, and S. B. Christman, Phys. Rev. B 33, 7906 (1986).

[19] H.-J. Ernst, E. Hulpke, and J. P. Toennies, Phys. Rev. B 46, 16081 (1992).

[20] W. K. Han, D. Sahu, and S. C. Ying, Phys. Rev. B 41, 4403 (1990).

[21] G. Y. Hu and S. C. Ying, Physica (Amsterdam) 140A, 585 (1987).

[22] A. Cucchetti and S. C. Ying, Phys. Rev. B 54, 3300 (1996).

[23] E. Falck, M.S. thesis, Helsinki University of Technology, 2001.

[24] M. E. Fisher and M. N. Barber, Phys. Rev. Lett. 28, 1516 (1972).

[25] E. A. Daniels and R. Gomer, Surf. Sci. 336, 245 (1995).

[26] The diffusion coefficient away from the transition region has weak coverage dependence in both the present study and from Ref. [25]. The only exception is for the $0.12 \mathrm{ML}$ coverage where the data from Ref. [25] shows a strong anisotropy, with the diffusion along the [010] direction considerably higher than all other cases. The origin for this anisotropy is not understood.

[27] F. Hofmann, J. R. Manson, and J.P. Toennies, J. Chem. Phys. 101, 10155 (1994).

[28] E. Granato, T. Ala-Nissila, and S. C. Ying, Phys. Rev. B 62, 11834 (2000).

[29] X. S. Ling, S. R. Park, B. A. McClain, S. M. Choi, D. C. Dender, and J. W. Lynn, Phys. Rev. Lett. 86, 712 (2001). 